T. OKAYASU

KODAI MATH. J.

10 (1987), $116-126$

\title{
MINIMAL IMMERSIONS OF CURVATURE PINCHED 2-MANIFOLDS INTO SPHERES
}

\author{
By TAKASHI OKAYASU
}

\section{Introduction.}

Let $(M, g)$ be a closed, connected two-dimensional Riemannian manifold. We consider isometric minimal immersions $\phi: M \rightarrow S^{N}(1)$ into the $N$-dimensional unit sphere of the Euclidean space $R^{N+1}$. Let $S^{2}(K)$ be a sphere of constant curvature $K$ and let $s \in N, K(s)=2[s(s+1)]^{-1}$. In [3] Borůvka constructed isometric minimal immersions $\psi_{s}: S^{2}(K(s)) \rightarrow S^{2 s}(1)$. Later Calabi proved that any isometric minimal full immersion of $S^{2}(K)$ into $S^{N}(1)$ is congruent to some $\Psi_{s}$ ([5]).

Because of Calabi's result Simon cojectured the following ([6]).

Conjecture. Let $(M, g)$ be a closed, connected 2-manifold with curvature $K$. Let $s \in N$ and let $\phi: M \rightarrow S^{N}(1)$ be an isometric minimal immersion such that $K(s+1) \leqq K \leqq K(s)$. Then either $K \equiv K(s)$ or $K \equiv K(s+1)$ on $(M, g)$ and $\phi=\phi_{s}$ or $\phi=\phi_{s+1}$, respectively.

The conjecture is true for $s=1$ and $s=2$ (cf. [7] for $s=1$ and $N=4$; [2] for $s=1, N$ arbitrary ; [6], [8] for $s=2$ ).

In this paper we give a partial positive answer to this conjecture for $s=3$; we prove:

THEOREM. For a real number $K_{0}$ satisfying $1 / 10<K_{0}<1 / 6$, put $K_{1}=$ $K_{0}+(1 / 18)\left(1-6 K_{0}\right)\left(10 K_{0}-1\right)$ (then $\left.1 / 10<K_{0}<K_{1}<1 / 6\right)$. Let $(M, g)$ be a closed, connected 2-dimensional Riemannian manifold with curvature $K$. Assume that $K_{0} \leqq K \leqq K_{1}$. Then there exists no isometric minimal immersion $\phi: M \rightarrow S^{N}(1)$ for any $N$.

In the course of the proof, we also get short proofs for the case $s=1,2$.

The author would like to express hearty thanks to Professor S. Tanno for his advice and encouragement during the developement of this work.

Received October 14, 1986 


\section{Proof.}

The main idea of our proof is to use the operators $X, Y$ and $H$, which were introduced by Bryant for classifying minimal surfaces with constant Gaussian curvature in spheres ([4]), in our variable curvature case.

We describe the definitions of the operators $X, Y$ and $H$ following Bryant ([4]). Let $\left(M^{2}, g\right)$ be an oriented, connected 2-dimensional Riemannian manifold. We let $\chi: \mathscr{T} \rightarrow M$ be the bundle of oriented or thonormal frames. Thus $f \in \mathscr{F}$ is a triple $f=\left(x, e_{1}, e_{2}\right)$, where $x \in M$ and $e_{1}, e_{2} \in T_{x} M$ form an oriented basis. The canonical 1-forms, $\omega^{1}, \omega^{2}$ on $\mathscr{F}$ are the unique 1-forms satisfying $d \chi=e_{1} \omega^{1}+e_{2} \omega^{2}$. Set $\omega=\omega^{1}+i \omega^{2}, \quad \bar{\omega}=\omega^{1}-i \omega^{2}$. Let $\tau \rightarrow M$ be the complex line bundle of 1-forms which are multiples of $\omega$ and let $\tau^{-1} \rightarrow M$ be the complex line bundle of 1 -forms which are multiples of $\bar{\omega}$. For $m \geqq 0$, let $\tau^{m} \rightarrow M\left(\right.$ resp. $\tau^{-m} \rightarrow M$ ) be the $m$-th power of $\tau \rightarrow M$ (resp. $\tau^{-1} \rightarrow M$ ) as a complex line bundle. Using the identification $\omega^{m}=(\bar{\omega})^{-m}$ for all $m$, we have a canonical pairing $\tau^{m} \times \tau^{k} \rightarrow \tau^{m+k}$ for all $m$ and $k$. Let $C^{\infty}\left(\tau^{m}\right)$ be the vector space consisting of all smooth sections of $\tau^{m}$. If $\sigma \in C^{\infty}\left(\tau^{m}\right)$, then, on $\mathscr{F}$, we may write $\sigma=s(\omega)^{m}$ for a unique function $s$ on $\mathscr{I}$. One easily computes that $d s=-m i \rho s+s^{\prime} \omega+s^{\prime \prime} \bar{\omega}$ for some unique functions $s^{\prime}$ and $s^{\prime \prime}$ on $\mathscr{F}$, where $\rho$ is the commection form. It is easy to see that the forms $s^{\prime}(\omega)^{m+1}=\sigma^{\prime}$ and $s^{\prime \prime}(\omega)^{m-1}=\sigma^{\prime \prime}$ are well-defined sections of $\tau^{m+1}$ and $\tau^{m-1}$ respectively. This allows us to define operators $\partial_{m}: C^{\infty}\left(\tau^{m}\right) \rightarrow$ $C^{\infty}\left(\tau^{m+1}\right)$ and $\bar{\partial}_{m}: C^{\infty}\left(\tau^{m}\right) \rightarrow C^{\infty}\left(\tau^{m-1}\right)$ by $\partial_{m} \sigma=\sigma^{\prime}, \bar{\partial}_{m} \sigma=\sigma^{\prime \prime}$. Let $I_{m}: C^{\infty}\left(\tau^{m}\right) \rightarrow C^{\infty}\left(\tau^{m}\right)$ be the identity map. Set $I=\bigoplus_{m} C^{\infty}\left(\tau^{m}\right)$ as a $Z$-graded vector space and define the operators

$$
X=\oplus_{m} \partial_{m}, \quad Y=\bigoplus_{m} \bar{\partial}_{m}, \quad H=\bigoplus_{m} m \cdot I_{m} .
$$

Thus for a function $f$ on $M$ regarded as a cross section of $\tau^{0}$, we get

$$
X f=\frac{1}{2}\left(e_{1} f-i e_{2} f\right) \omega, \quad Y f=\frac{1}{2}\left(e_{1} f+i e_{2} f\right) \bar{\omega} .
$$

Let $\langle$,$\rangle denote the standard inner product on R^{N+1}$. We set $\mathcal{} \mathcal{V}=R^{N+1} \otimes_{R} \mathscr{I}$ and extend the operators $X, Y$ and $H$ to $\mathcal{V}$ in the natural way. We also have a pairing $\langle\rangle:, \mathcal{Q} \times \mathcal{Q} \rightarrow \mathscr{I}$ extending the given $\langle$,$\rangle in the obvious fashion. We$ define conjugation in $\mathcal{V}$ by setting $\bar{\sigma}=\bar{s}(\omega)^{-m}$ for $\sigma=s(\omega)^{m} \in C^{\infty}\left(\tau^{m}\right)$. Thus we have $X \bar{\sigma}=\overline{Y \sigma}, Y \bar{\sigma}=\overline{X \sigma}$.

We have the following two propositions (see Proposition 1.1 and Proposition 1.2 in [4]).

Proposition 1. The operators $X, Y$ and $H$ satisfy

$$
\begin{aligned}
& {[X, Y]=\left(-\frac{K}{2}\right) H,} \\
& \Delta=2(X Y+Y X),
\end{aligned}
$$

where $K$ is the Gaussian curvature of $M$ and $\Delta: \mathcal{I} \rightarrow \mathscr{I}$ is the Laplace-Beltrami 
operator on each graded piece.

Proposition 2. Let $\phi: M^{2} \rightarrow S^{N}(1) \subset R^{N+1}$ be an isometric minimal immersion of an oriented 2-dimensional Riemannian manifold $M$. Then

$$
\langle\phi, \phi\rangle=1 ;
$$$$
\langle X \phi, X \phi\rangle=0, \quad\langle Y \phi, Y \phi\rangle=0 \text {; }
$$$$
\langle X \phi, Y \phi\rangle=\frac{1}{2}
$$$$
\Delta \phi=-2 \phi \text {. }
$$

LEMMA 1. Let $\left(M^{2}, g\right)$ be an oriented 2-dimensional Riemannian manifold. If $f$ is a smooth function on $M$, then

$$
\Delta f=4 X Y f=4 Y X f .
$$

If $f, h$ are smooth functions on $M$, then

$$
X f \cdot Y h+Y f \cdot X h=\frac{1}{2} g(\operatorname{grad} f, \operatorname{grad} h) .
$$

Proof. Since $f \in \mathcal{I}$ has degree $0, H f=0$. Thus

$$
\Delta f=2(X Y f+Y X f), \quad(X Y-Y X) f==-\frac{K}{2} H f=0,
$$

from which (8) follows. From (1) we obtain

$$
X f \cdot Y h=\frac{1}{4}\left(e_{1} f-\imath e_{2} f\right)\left(e_{1} h+\imath e_{2} h\right) .
$$

Therefore

$$
X f \cdot Y h+Y f \cdot X h=\frac{1}{2}\left(e_{1} f \cdot e_{1} h+e_{2} f \cdot e_{2} h\right),
$$

from which (9) follows.

LEMMA 2. Let $\phi: M^{2} \rightarrow S^{N}(1) \subset R^{N+1}$ be an isometric minumal immersion of an oriented 2-dimensional Riemannian manifold. Then

$$
\begin{aligned}
& X Y \phi=Y X \phi=-\frac{1}{2} f ; \\
& \langle\phi, X \phi\rangle=0, \quad\langle\phi, Y \phi\rangle=0 ; \\
& \left\langle X \phi, Y^{2} \phi\right\rangle=0, \quad\left\langle Y \phi, X^{2} \phi\right\rangle=0 ; \\
& X Y^{2} \phi=\frac{K-1}{2} Y \phi, \quad Y X^{2} \phi=\frac{K-1}{2} X \phi ;
\end{aligned}
$$




$$
\begin{aligned}
& \left\langle X^{2} \phi, Y^{2} \phi\right\rangle=\frac{1-K}{4} \\
& \left\langle X^{2} \phi, Y^{3} \phi\right\rangle=-\frac{1}{4} Y K, \quad\left\langle Y^{2} \phi, X^{3} \phi\right\rangle=-\frac{1}{4} X K
\end{aligned}
$$$$
X Y^{3} \phi=\frac{1}{2} Y K \cdot Y \phi+\frac{3 K-1}{2} Y^{2} \phi,
$$$$
Y X^{3} \phi=\frac{1}{2} X K \cdot X \phi+\frac{3 K-1}{2} X^{2} \phi ;
$$

$\left\langle X \phi, Y^{3} \phi\right\rangle=0, \quad\left\langle Y \phi, X^{3} \phi\right\rangle=0 ;$

$$
\left\langle X^{3} \phi, Y^{3} \phi\right\rangle=\frac{(1-K)(1-3 K)}{8}-\frac{\Delta K}{16} ;
$$$$
\left\langle X^{3} \phi, Y^{4} \phi\right\rangle=\frac{9 K-5}{8} Y K-\frac{Y(\Delta K)}{16},
$$$$
\left\langle Y^{3} \phi, X^{4} \phi\right\rangle=\frac{9 K-5}{8} X K-\frac{X(\Delta K)}{16} ;
$$$$
X Y^{4} \phi=\frac{1}{2} Y^{2} K \cdot Y \phi+2 Y K \cdot Y^{2} \phi+\frac{6 K-1}{2} Y^{3} \phi,
$$$$
Y X^{4} \phi=\frac{1}{2} X^{2} K \cdot X \phi+2 X K \cdot X^{2} \phi+\frac{6 K-1}{2} X^{3} \phi ;
$$

$\left\langle X^{4} \phi, Y^{4} \phi\right\rangle=\frac{(1-K)(1-3 K)(1-6 K)}{16}-\frac{1}{16}\|\operatorname{grad} K\|^{2}$

$$
+\frac{15}{64} \Delta K^{\mathrm{2}}-\frac{3}{16} \Delta K-\frac{1}{64} \Delta(\Delta K) \text {. }
$$

Proof. Applying Lemma 1 to each component of $\phi$ we obtain $\Delta \phi=4 X Y \phi$ $=4 Y X \phi$. Then (10) follows immediately from (7). Operating $X$ to $\langle\phi, \phi\rangle=1$, we get $\langle\phi, X \phi\rangle=0$. Since $\bar{\phi}=\phi$, we get $\langle\phi, Y \phi\rangle=0$. Operating $Y$ to (6), we get $\langle Y X \phi, Y \phi\rangle+\left\langle X \phi, Y^{2} \phi\right\rangle=0$. By (10) and (11) $\left\langle X \phi, Y^{2} \phi\right\rangle$ vanishes. The second equation of (12) is the conjugate of the first. By (2) and (10) we get

$$
\begin{aligned}
X Y^{2} \phi & =(X Y)(Y \phi)=\left(Y X-\frac{K}{2} H\right)(Y \phi)=Y(X Y \phi)+\frac{K}{2} Y \phi \\
& =-\frac{1}{2} Y \phi+\frac{K}{2} Y \phi=\frac{K-1}{2} Y \phi
\end{aligned}
$$

which proves (13). Operating $X$ to $\left\langle X \phi, Y^{2} \phi\right\rangle=0$ and using (13) and (6), we get 


$$
\left\langle X^{2} \phi, Y^{2} \phi\right\rangle=-\left\langle X \phi, X Y^{2} \phi\right\rangle=-\frac{K-1}{2}\langle X \phi, Y \phi\rangle=\frac{1-K}{4},
$$

which proves (14). Operating $Y$ to (14) and using (13) and (12), we get

$$
-\frac{1}{4} Y K=\left\langle Y X^{2} \phi, Y^{2} \phi\right\rangle+\left\langle X^{2} \phi, Y^{3} \phi\right\rangle=\left\langle X^{2} \phi, Y^{3} \phi\right\rangle,
$$

which proves (15). By (2) and (13) we get

$$
\begin{aligned}
X Y^{3} \phi & =X Y\left(Y^{2} \phi\right)=\left(Y X-\frac{K}{2} H\right)\left(Y^{2} \phi\right)=Y\left(X Y^{2} \phi\right)+K \cdot Y^{2} \phi \\
& =Y\left(\frac{K-1}{2} Y \phi\right)+K \cdot Y^{2} \phi=\frac{Y K}{2} Y \phi+\frac{3 K-1}{2} Y^{2} \phi
\end{aligned}
$$

which proves (16). Operating $Y$ to $\left\langle X \phi, Y^{2} \phi\right\rangle=0$ and using (10), (11) and (5), we get

$$
\begin{aligned}
\left\langle X \phi, Y^{3} \phi\right\rangle & =-\left\langle Y X \phi, Y^{2} \phi\right\rangle=\frac{1}{2}\left\langle\phi, Y^{2} \phi\right\rangle \\
& =\frac{1}{2} Y\langle\phi, Y \phi\rangle-\frac{1}{2}\langle Y \phi, Y \phi\rangle=0,
\end{aligned}
$$

which proves (17). Operating $X$ to $\left\langle X^{2} \phi, Y^{3} \phi\right\rangle=-(1 / 4) Y K$ and using (8), (16), (12) and (14), we get

$$
\begin{aligned}
\left\langle X^{3} \phi, Y^{3} \phi\right\rangle & =-\frac{1}{16} \Delta K-\left\langle X^{2} \phi, X Y^{3} \phi\right\rangle \\
& =-\frac{1}{16} \Delta K-\left\langle X^{2} \phi, \frac{1}{2} Y K \cdot Y \phi+\frac{3 K-1}{2} Y^{2} \phi\right\rangle \\
& =-\frac{1}{16} \Delta K+\frac{(1-K)(1-3 K)}{8},
\end{aligned}
$$

which proves (18). Operating $Y$ to (18) and using (16), (17) and (15), we get

$$
\begin{aligned}
\left\langle X^{3} \phi, Y^{4} \phi\right\rangle & =-\frac{1}{16} Y(\Delta K)+\frac{3 K-2}{4} Y K-\left\langle Y X^{3} \phi, Y^{3} \phi\right\rangle \\
& =-\frac{1}{16} Y(\Delta K)+\frac{9 K-5}{8} Y K,
\end{aligned}
$$

which proves (19). By (2) and (16) we get

$$
\begin{aligned}
X Y^{4} \phi & =\left(Y X-\frac{K}{2} H\right)\left(Y^{3} \phi\right)=Y\left(X Y^{3} \phi\right)+\frac{3 K}{2} Y^{3} \phi \\
& =\frac{1}{2} Y^{2} K \cdot Y \phi+2 Y K \cdot Y^{2} \phi+\frac{6 K-1}{2} Y^{3} \phi .
\end{aligned}
$$


This proves (20). Operating $X$ to the first equation of (19) and using (8), (9) and (20), we get

$$
\begin{aligned}
\left\langle X^{4} \phi, Y^{4} \phi\right\rangle & =\frac{9}{32}\|\operatorname{grad} K\|^{2}+\frac{9 K-5}{32} \Delta K-\frac{1}{64} \Delta(\Delta K)-\left\langle X^{3} \phi, X Y^{4} \phi\right\rangle \\
& =\frac{9}{32}\|\operatorname{grad} K\|^{2}+\frac{9 K-5}{32} \Delta K-\frac{1}{64} \Delta(\Delta K) \\
& -\left\langle X^{3} \phi, \frac{1}{2} Y^{2} K \cdot Y \phi+2 Y K \cdot Y^{2} \phi+\frac{6 K-1}{2} Y^{3} \phi\right\rangle .
\end{aligned}
$$

By using (17), (15), (18) and $K \Delta K=(1 / 2) \Delta K^{2}-\|\operatorname{grad} K\|^{2}$, (21) follows easily from the last equation.

Now we can give proofs for the case $s=1,2$. We briefly explain the case $s=2$. Let $(M, g)$ be a closed 2-dimensional Riemannian manifold with $1 / 6 \leqq K$ $\leqq 1 / 3$. Let $\Phi: M \rightarrow S^{N}(1) \subset R^{N+1}$ be an isometric minimal immersion. We may assume $M$ is orientable. Integration of (21) gives

$$
\int_{M}\left\{\left\langle X^{4} \phi, Y^{4} \phi\right\rangle+\frac{(1-K)(1-3 K)(6 K-1)}{16}+\frac{1}{16}\|\operatorname{grad} K\|^{2}\right\}=0 .
$$

Since $\left\langle X^{4} \phi, Y^{4} \phi\right\rangle=\left\langle X^{4} \phi, \overline{X^{4} \phi}\right\rangle \geqq 0$ and $1 / 6 \leqq K \leqq 1 / 3$, the integrands on the left hand side are all non-negative. Therefore $K \equiv 1 / 3$ or $1 / 6$. Thus we get the conclusion from Calabi's theorem ([5]).

From now on we assume that $\phi: M \rightarrow S^{N}(1) \subset R^{N+1}$ is an isometric minimal immersion of a closed, connected 2-dimensional Riemannian manifold $M$. We may assume that $M$ is orientable.

Set $\left\langle X^{4} \phi, Y^{4} \phi\right\rangle=F$. Operating $Y$ to $\left\langle X^{4} \phi, Y^{4} \phi\right\rangle=F$ and using (20), we get

$$
\begin{aligned}
\left\langle X^{4} \phi, Y^{5} \phi\right\rangle= & Y F-\left\langle Y X^{4} \phi, Y^{4} \phi\right\rangle \\
= & Y F-\frac{X^{2} K}{2} \cdot\left\langle X \phi, Y^{4} \phi\right\rangle-2 X K\left\langle X^{2} \phi, Y^{4} \phi\right\rangle \\
& -\frac{6 K-1}{2} \cdot\left\langle X^{3} \phi, Y^{4} \phi\right\rangle .
\end{aligned}
$$

On the other hand, by (17), (10), (11) and (5), we get

$$
\begin{aligned}
\left\langle X \phi, Y^{4} \phi\right\rangle & =-\left\langle Y X \phi, Y^{3} \phi\right\rangle=\frac{1}{2}\left\langle\phi, Y^{3} \phi\right\rangle \\
& =\frac{1}{2} Y\left\langle\phi, Y^{2} \phi\right\rangle-\frac{1}{2}\left\langle Y \phi, Y^{2} \phi\right\rangle \\
& =\frac{1}{2} Y\{Y\langle\phi, Y \phi\rangle-\langle Y \phi, Y \phi\rangle\}-\frac{1}{4} Y\langle Y \phi, Y \phi\rangle \\
& =0 .
\end{aligned}
$$


By (15), (13), (17) and (9), we get

$$
\begin{aligned}
X K\left\langle X^{2} \dot{\phi}, Y^{4} \phi\right\rangle & =X K\left\{Y\left\langle X^{2} \phi, Y^{3} \phi\right\rangle-\left\langle Y X^{2} \phi, Y^{3} \phi\right\rangle\right\} \\
& =X K\left\{-\frac{1}{4} Y^{2} K-\frac{K-1}{2}\left\langle X \phi, Y^{3} \phi\right\rangle\right\} \\
& =-\frac{1}{4} X K \cdot Y^{2} K \\
& =-\frac{1}{4}\{Y(X K \cdot Y K)-Y X K \cdot Y K\} \\
& =-\frac{1}{16} Y\left(\|\operatorname{grad} K\|^{2}\right)+\frac{1}{16} \Delta K \cdot Y K
\end{aligned}
$$

Substituting (23), (24) and (19) to (22), we obtain

$$
\begin{aligned}
\left\langle X^{4} \phi, Y^{5} \phi\right\rangle= & Y H+Y\left(\frac{1}{8}\|\operatorname{grad} K\|^{2}\right)-\frac{1}{8} \Delta K \cdot Y K \\
& -\frac{(6 K-1)(9 K-5)}{16} Y K+\frac{6 K-1}{32} Y(\Delta K) .
\end{aligned}
$$

Operating $X$ to (25), we get

$$
\begin{aligned}
\left\langle X^{5} \phi,\right. & \left.Y^{5} \phi\right\rangle+\left\langle X^{4} \phi, X Y^{5} \phi\right\rangle \\
= & X Y F+\frac{1}{8} X Y\left(\|\operatorname{grad} K\|^{2}\right)-\frac{1}{8} X(\Delta K \cdot Y K) \\
& -\frac{1}{16} X\{(6 K-1)(9 K-5) Y K\}+\frac{1}{32} X\{(6 K-1) Y(\Delta K)\} .
\end{aligned}
$$

Adding (26) to its conjugate, we get

$$
\begin{aligned}
2\left\langle X^{5} \phi,\right. & \left.Y^{5} \phi\right\rangle+\left\langle X^{4} \phi, X Y^{5} \phi\right\rangle+\left\langle Y^{4} \phi, Y X^{5} \phi\right\rangle \\
= & \frac{1}{2} \Delta F+\frac{1}{16} \Delta\left(\|\operatorname{grad} K\|^{2}\right)-\frac{1}{8}\{X(\Delta K \cdot Y K)+Y(\Delta K \cdot X K)\} \\
& -\frac{1}{16}\{X\{(6 K-1)(9 K-5) Y K\}+Y\{(6 K-1)(9 K-5) X K\}\} \\
& +\frac{1}{32}\{X\{(6 K-1) Y(\Delta K)\}+Y\{(6 K-1) X(\Delta K)\}\} .
\end{aligned}
$$

We need the following lemma.

LEMMA 3. Let $\left(M^{\prime}, g^{\prime}\right)$ be a closed, orientable 2-dimensional Riemannian manifold and $f, h$ be functions on $M^{\prime}$. Then

$$
\int_{M^{\prime}}\{X(f \cdot Y h)+Y(f \cdot X h)\}=0 .
$$


Proof. By using (9) and Green's formula, we get

$$
\begin{aligned}
& \int_{M^{\prime}}\{X(f \cdot Y h)+Y(f \cdot X h)\} \\
= & \int_{M^{\prime}}\{X f \cdot Y h+Y f \cdot X h+f \cdot X Y h+f \cdot Y X h\} \\
= & \int_{M^{\prime}}\left\{\frac{1}{2} g^{\prime}(\operatorname{grad} f, \operatorname{grad} h)+\frac{1}{2} f \cdot \Delta h\right\}=0 .
\end{aligned}
$$

We integrate (27) and apply Lemma 3. Then we get (28)

$$
\int_{M^{\prime}}\left\{2\left\langle X^{5} \phi, Y^{5} \phi\right\rangle+\left\langle X^{4} \phi, X Y^{5} \phi\right\rangle+\left\langle Y^{4} \phi, Y X^{5} \phi\right\rangle\right\}=0 .
$$

We compute $X Y^{5} \phi$. By (20) we get

$$
\begin{aligned}
X Y^{5} \phi & =\left(Y X-\frac{K}{2} H\right)\left(Y^{4} \phi\right)=Y\left(X Y^{4} \phi\right)+2 K \cdot Y^{4} \phi \\
& =Y\left\{\frac{1}{2} Y^{2} K \cdot Y \phi+2 Y K \cdot Y^{2} \phi+\frac{6 K-1}{2} Y^{3} \phi\right\}+2 K \cdot Y^{4} \Phi \\
& =\frac{1}{2} Y^{3} K \cdot Y \phi+\frac{5}{2} Y^{2} K \cdot Y^{2} \phi+5 Y K \cdot Y^{3} \phi+\frac{10 K-1}{2} Y^{4} \phi
\end{aligned}
$$

By (29), (23), (19), (15) and (17), we get

$$
\begin{aligned}
\left\langle X^{4} \phi, X Y^{5} \phi\right\rangle= & \frac{1}{2} Y^{3} K\left\langle X^{4} \phi, Y \phi\right\rangle+\frac{5}{2} Y^{2} K\left\langle X^{4} \phi, Y^{2} \phi\right\rangle \\
& +5 Y K\left\langle X^{4} \phi, Y^{3} \phi\right\rangle+\frac{10 K-1}{2}\left\langle X^{4} \phi, Y^{4} \phi\right\rangle \\
= & \frac{5}{2} Y^{2} K\left\{X\left\langle X^{3} \phi, Y^{2} \phi\right\rangle-\left\langle X^{3} \phi, X Y^{2} \phi\right\rangle\right\} \\
& +5 Y K\left\{\frac{9 K-5}{8} X K-\frac{1}{16} X(\Delta K)\right\}+\frac{10 K-1}{2}\left\langle X^{4} \phi, Y^{4} \phi\right\rangle \\
= & -\frac{5}{8} Y^{2} K \cdot X^{2} K+\frac{5(9 K-5)}{32}\|\operatorname{grad} K\|^{2} \\
& -\frac{5}{16} Y K \cdot X(\Delta K)+\frac{10 K-1}{2}\left\langle X^{4} \phi, Y^{4} \phi\right\rangle .
\end{aligned}
$$

From (28) and (30), we get

$$
\begin{aligned}
\int_{M}\left\{2 \left\langleX^{5} \Phi,\right.\right. & \left.Y^{5} \Phi\right\rangle-\frac{5}{4} X^{2} K \cdot Y^{2} K+\frac{5(9 K-5)}{16}\|\operatorname{grad} K\|^{2} \\
& \left.-\frac{5}{32} g(\operatorname{grad} K, \operatorname{grad}(\Delta K))+(10 K-1)\left\langle X^{4} \phi, Y^{4} \phi\right\rangle\right\} \\
= & 0 .
\end{aligned}
$$


On the other hand, we have

$$
\begin{aligned}
& \int_{M} X^{2} K \cdot Y^{2} K=\int_{M}\left\{X\left(X K \cdot Y^{2} K\right)-X K \cdot X Y^{2} K\right\} \\
= & \int_{M}\left\{X\{Y(X K \cdot Y K)-Y X K \cdot Y K\}-X K \cdot\left(Y X-\frac{K}{2} H\right)(Y K)\right\} \\
= & \int_{M}\left\{\frac{1}{16} \Delta\|\operatorname{grad} K\|^{2}-\frac{1}{4} X(\Delta K \cdot Y K)-\frac{1}{4} X K \cdot Y(\Delta K)-\frac{K}{2} X K \cdot Y K\right\} \\
= & \int_{M}\left\{-\frac{1}{4}\{X(\Delta K) \cdot Y K+Y(\Delta K) \cdot X K\}-\frac{1}{16}(\Delta K)^{2}-\frac{K}{8}\|\operatorname{grad} K\|^{2}\right\} \\
= & \int_{M}\left\{-\frac{1}{8} g\left\{(\operatorname{grad} \Delta K, \operatorname{grad} K)-\frac{1}{16}(\Delta K)^{2}-\frac{1}{8} K\|\operatorname{grad} K\|^{2}\right\}\right. \\
= & \int_{M}\left\{\frac{1}{16}(\Delta K)^{2}-\frac{K}{8}\|\operatorname{grad} K\|^{2}\right\} .
\end{aligned}
$$

From (31) and (32), we get

$$
\begin{aligned}
\int_{M}\left\{2\left\langle X^{5} \phi, Y^{5} \phi\right\rangle\right. & +\frac{5}{64}(\Delta K)^{2}+\frac{95 K-50}{32}\|\operatorname{grad} K\|^{2} \\
& \left.+(10 K-1)\left\langle X^{4}, \phi Y^{4} \phi\right\rangle\right\}=0 .
\end{aligned}
$$

By (21) we get from (33)

$$
\begin{aligned}
0= & \int_{M}\left\{2\left\langle X^{5} \Phi, Y^{5} \Phi\right\rangle+\frac{5}{64}(\Delta K)^{2}+\frac{95 K-50}{32}\|\operatorname{grad} K\|^{2}\right. \\
& +\frac{10 K-1}{2}\left\langle X^{4} \Phi, Y^{4} \Phi\right\rangle+\frac{10 K-1}{2}\left[\frac{(1-K)(1-3 K)(1-6 K)}{16}\right. \\
& \left.\left.-\frac{1}{16}\|\operatorname{grad} K\|^{2}+\frac{15}{64} \Delta K^{2}-\frac{3}{16} \Delta K-\frac{1}{64} \Delta(\Delta K)\right]\right\} \\
= & \int_{M}\left\{2\left\langle X^{5} \phi, Y^{5} \phi\right\rangle+\frac{1}{32}(1-K)(1-3 K)(1-6 K)(10 K-1)\right. \\
& \left.+\frac{10 K-1}{2}\left\langle X^{4} \Phi, Y^{4} \Phi\right\rangle+\frac{10 K-19}{32}\|\operatorname{grad} K\|^{2}\right\} .
\end{aligned}
$$

Let $K_{0}, K_{1}$ be constants. Then from (34) and (21) we get

$$
\begin{aligned}
0= & \int_{M}\left\{2\left\langle X^{5} \phi, Y^{5} \phi\right\rangle+\frac{1}{32}(1-K)(1-3 K)(1-6 K)(10 K-1)\right. \\
& +\frac{10\left(K-K_{0}\right)}{2}\left\langle X^{4} \phi, Y^{4} \phi\right\rangle+\frac{10 K_{0}-1}{2}\left\langle X^{4} \phi, Y^{4} \phi\right\rangle \\
& \left.+\frac{10\left(K-K_{0}\right)}{32}\|\operatorname{grad} K\|^{2}+\frac{10 K_{0}-10}{32}\|\operatorname{grad} K\|^{2}\right\}
\end{aligned}
$$




$$
\begin{aligned}
= & \int_{M}\left\{2\left\langle X^{5} \phi, Y^{5} \phi\right\rangle+\frac{1}{32}(1-K)(1-3 K)(1-6 K)\left(10 K-1+10 K_{0}-1\right)\right. \\
& \left.+5\left(K-K_{0}\right)\left\langle X^{4} \phi, Y^{4} \phi\right\rangle+\frac{5}{16}\left(K-K_{0}\right)\|\operatorname{grad} K\|^{2}-\frac{9}{16}\|\operatorname{grad} K\|^{2}\right\} .
\end{aligned}
$$

On the other hand, by (18) we have

$$
\begin{aligned}
& \int_{M}\left\{-\frac{9}{16}\|\operatorname{grad} K\|^{2}\right\}=\int_{M} \frac{9}{16} K \Delta K=\int_{M} \frac{9}{16}\left(K-K_{1}\right) \Delta K \\
= & \int_{M} 9\left(K_{1}-K\right)\left\{\left\langle X^{3} \phi, Y^{3} \phi\right\rangle-\frac{1}{8}(1-K)(1-3 K)\right\} \\
= & \int_{M}\left\{9\left(K_{1}-K\right)\left\langle X^{3} \phi, Y^{3} \phi\right\rangle-\frac{9}{8}(1-K)(1-3 K)\left(K_{1}-K\right)\right\} .
\end{aligned}
$$

From (35) and (36) we get

$$
\begin{aligned}
0= & \int_{M}\left\{2\left\langle X^{5} \phi, Y^{5} \phi\right\rangle+5\left(K-K_{0}\right)\left\langle X^{4} \phi, Y^{4} \phi\right\rangle\right. \\
& +\frac{5}{16}\left(K-K_{0}\right)\|\operatorname{grad} K\|^{2}+9\left(K_{1}-K\right)\left\langle X^{3} \phi, Y^{3} \phi\right\rangle \\
& \left.+\frac{1}{32}(1-K)(1-3 K)\left[(1-6 K)\left(10 K+10 K_{0}-2\right)+36\left(K-K_{1}\right)\right]\right\} .
\end{aligned}
$$

Let $1 / 10<K_{0}<1 / 6$ and put $K_{1}=K_{0}+(1 / 18)\left(1-6 K_{0}\right)\left(10 K_{0}-1\right)$. Then $1 / 10<K_{0}$ $<K_{1}<1 / 6$. We define $h(t)=(1-6 t)\left(10 t+10 K_{0}-2\right)+36\left(t-K_{1}\right)$. Then $h\left(K_{0}\right)=0$ and $h\left(K_{1}\right)>0$. Thus $h(K)>0$ for $K_{0}<K \leqq K_{1}$.

We can rewrite (37) as follows.

$$
\begin{aligned}
0= & \int_{M}\left\{2\left\langle X^{5} \phi, Y^{5} \phi\right\rangle+5\left(K-K_{0}\right)\left\langle X^{4} \phi, Y^{4} \phi\right\rangle\right. \\
& +\frac{5}{16}\left(K-K_{0}\right)\|\operatorname{grad} K\|^{2}+9\left(K_{1}-K\right)\left\langle X^{3} \phi, Y^{3} \phi\right\rangle \\
& \left.+\frac{1}{32}(1-K)(1-3 K) h(K)\right\} .
\end{aligned}
$$

The integrands of the right hand side are all non-negative under the assumption of the theorem. Therefore $(1-K)(1-3 K) h(K) \equiv 0$. That is $K \equiv K_{0}$. Since $1 / 10<K_{0}<1 / 6$, according to Calabi's theorem ([4]), this is impossible. This completes the proof of the theorem.

(q. e. d.).

Remarks. (1) Since $K_{1}-K_{0}=(1 / 18)\left(1-6 K_{0}\right)\left(10 K_{0}-1\right)=(1 / 18)\left\{-60\left(K_{0}-2 / 15\right)^{2}\right.$ $+1 / 15\}, \max \left(K_{1}-K_{0}\right)=1 /(18 \cdot 15)$. This value is $1 / 18$ of $1 / 6-1 / 10$.

(2) At present we need some additional assumption to prove the conjecture for $s \geqq 3([1],[9])$. 


\section{REFERENCES}

[1] A.C. Asperti, A note on the minimal immersions of the two-sphere, preprint.

[2] K. Benko, M. Kothe, K. D. Semmler and U. Simon, Eigenvalues of the Laplacian and curvature, Colloq. Math., 42 (1979), 19-31.

[3] O. BORŮVKA, Sur les surfaces représentées par les fonctions sphèriques de premiere espèce, J. Math. Pure et Appl., 12 (1933), 337-383.

[4] R.L. BRYANT, Minimal surfaces of constant curvature in $S^{n}$, Trans. Amer. Math., 290 (1985), 259-271.

[5] E. CALABI, Minimal immersions of surfaces in Euclidean spheres, J. Diff. Geom., 1 (1967), 111-125.

[6] M. Kozlowski and U. Simon, Minimal immersions of 2-manifolds into spheres, Math. Z., 186 (1984), 377-382.

[7] H. B. LAwSON, Local rigidity theorem for minimal hypersurfaces, Ann. Math., (2) 89 (1969), 187-197.

[8] T. Ogata, Minimal surfaces in a sphere with Gaussian curvature not less than 1/6, Tohoku Math. J., 37 (1985), 553-560.

[9] T. Ogata, U. Simon's conjecture on minimal surfaces in a sphere, preprint.

Department of Mathematics

FACULTY OF SCINCE

HiROSAKI UNIVERSITY

BUNKYO-CHO 3 HIROSAKI

AOMORI-KEN 036 JAPAN 